\title{
Using Competing Ant Colonies to Solve k-way Partitioning Problems with Foraging and Raiding Strategies
}

\author{
A. E. Langham ${ }^{\star}$ and P. W. Grant \\ Department of Computer Science \\ University of Wales Swansea \\ Singleton Park \\ Swansea, SA2 8PP, U.K \\ csjim,p.w.grant@swan.ac.uk
}

\begin{abstract}
The self organizing properties of ant colonies are employed to tackle the classical combinatorial optimization problem of graph partitioning. The graph is mapped onto an artificial environment in a manner that preserves the structural information. Ants from a number of colonies compete for resources. This leads to a restructuring of the global environment corresponding to a good partition. On the example graphs, this is shown to outperform the current best algorithms which are based on recursive bisection techniques.
\end{abstract}

\section{Introduction}

Simulation of fluid flow with the Finite Element Method approximates solution values across a domain using a set of discretized elements. In 2-Dimensional problems elements are planar polygons. Partitioning the domain for parallel processing can be mapped onto a graph partitioning problem where each vertex in the graph represents a node in the mesh, whilst an edge in the graph represents the need for communication between two nodes. The graph must be broken into approximately equal size sub-domains with as little communication between domains as possible.

There are graph partitioning problems which are NP-complete [5] and therefore we are looking for a near optimal partition in reasonable time. Most partitioning methods employ recursive bisection which can often provide a partition which is far from optimal [14] as regards minimizing the number of edge cuts. What seems optimal at the top level of recursion may provide a poor partition at lower levels given the benefit of hindsight. Recursive Spectral Bisection has been shown to be highly effective compared to alternative methods [13]. Multilevel implementations [8] are yet more effective especially in combination with local refinement using Kernighan Lin [9]. Recently many methods have been

\footnotetext{
* Supported by a University of Wales Validation Unit research studentship
} 
generalized to partition a graph into more than two sets at each stage of recursion [7]. It is thought that such methods could potentially produce better partitions. However, the direct computation of a good $\mathrm{k}$-way partitioning is harder than a bisection and hence recursive bisection is most commonly used. Dorigo et al. have applied the trail laying properties of ant colonies to the Travelling Salesman Problem, Quadratic Assignment Problem and Routing in telecommunication networks showing high quality results [3], [4], [1]. Kuntz et al. tackled the graph partitioning problem using a clustering algorithm [10] based on brood sorting in ant colonies [2] and a swarm colonization technique [11]. Such methods have shown good results on small graphs.

\section{The Approach}

Initially, we consider the bisection case in which two competing colonies of ants are used to split the graph into two partitions. Each colony is centred around a fixed cell in a grid which represents the environment in which the ants can navigate. The ants must learn to forage for food, each piece of food on the grid represents a node in the mesh which is being partitioned. The ants must find all the food and place it in the appropriate nest so that the set of nodes represented by the food in $N_{e s t}$ forms a set $V_{1}$ and the set of nodes in $N e s t_{2}$ forms a set $V_{2}$. The graph bisection problem for a graph $G=(V, E)$ with vertices $V$ and edges $E$, seeks a partition $V=V_{1} \cup V_{2}$ such that $V_{1} \cap V_{2}=\emptyset,\left|V_{1}\right| \approx\left|V_{2}\right|$ and the number of cut edges $\left|E_{c}\right|$ is minimized, where:

$$
E_{c}=\left\{\left(v_{1}, v_{2}\right) \in E \mid v_{1} \in V_{1}, v_{2} \in V_{2}\right\} .
$$

In order to cut down the search space we map the nodes onto the grid in a manner that represents the structure of the mesh by mapping the geometrical position of nodes in the mesh onto the equivalent geometrical position on the grid. To encourage the colonies to find a reasonable initial partition we place each nest at the center of an equal number of nodes using a recursive bisection heuristic. The main body of the loop, as shown below, is executed for each ant in each colony during every time step. The grid is initialized by placing the food and locating the nest for each colony. The body is iterated until there is no reduction in the number of cuts over 500 time steps. The best partition found during this time is taken to be the solution.

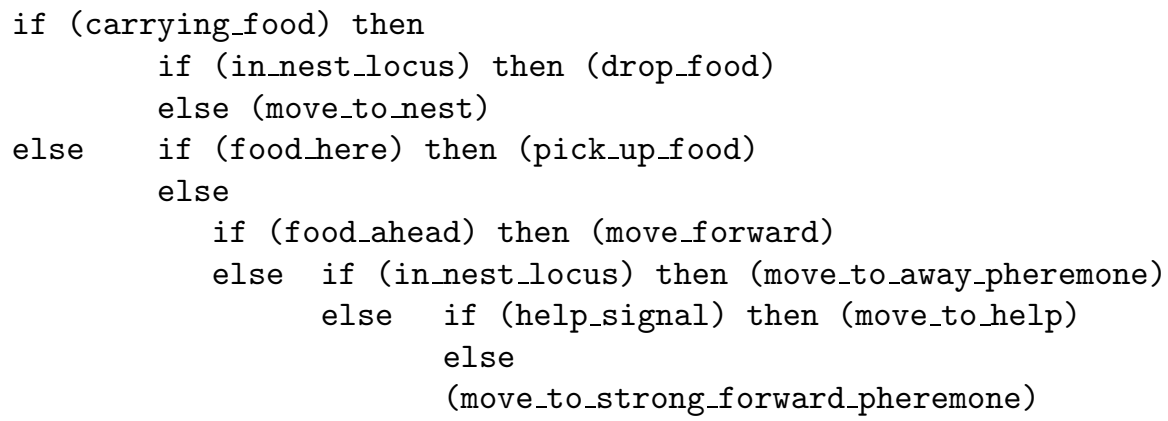


The actions and functions in this code are detailed below in Table 1. It should be noted that this foraging strategy can be generated using Genetic Program$\operatorname{ming}[12]$.

Table 1. Actions and Functions for Main Algorithm

\begin{tabular}{|c|c|}
\hline function/action & Explanation \\
\hline if_food_ahead & Return True if food is in the adjacent square. \\
\hline if_food_here & Return True if food is in the current square. \\
\hline if_in_nest_locus & $\begin{array}{l}\text { Return True if food is within two grid squares } \\
\text { of the colony nest. }\end{array}$ \\
\hline if_carrying_food & Return True if ant is carrying food. \\
\hline if_help_signal & $\begin{array}{l}\text { Return True if there is a help signal within a } \\
\text { locus of two grid squares. }\end{array}$ \\
\hline move_to_nest & $\begin{array}{l}\text { Move one step towards the nest in direction: } \\
\text { if }(\mid N \text { est. } x-x|\geq| N \text { est.y }-y \mid) \\
\text { then if }(x \geq N \text { est.x) then dir }=\text { WEST } \\
\text { else dir }=E A S T \\
\text { else if }(y \geq N \text { est.y) then dir }=\text { SOUTH } \\
\text { else dir }=\text { NORTH. }\end{array}$ \\
\hline move_to_help & Move one step towards the nearest help signal. \\
\hline move_to_away_pheremone & $\begin{array}{l}\text { Move one step away from the nest in the away } \\
\text { direction with the strongest pheremone trail. }\end{array}$ \\
\hline move_to_strong_forward_pheremone & $\begin{array}{l}\text { Move to an adjacent square with probability } \\
\text { dependent on the amount of pheremone present. }\end{array}$ \\
\hline pick_up_food ( foraging ) & $\begin{array}{l}\text { Food not belonging to a colony is picked up if } \\
\text { proportion of cuts created is less than half } \\
\text { total possible number. Number of ants needed is } \\
\text { related to the proportion of cuts. Help signal } \\
\text { sent out if not enough ants. }\end{array}$ \\
\hline pick_up_food ( raiding ) & $\begin{array}{l}\text { Food already in a colony is picked up with } \\
\text { probability } p \text { dependent on the change in the } \\
\text { proportion of cuts } \delta p_{c} \text { where } \\
p= \begin{cases}1.0 & \text { if } \delta p_{c}>0 \\
0.5 & \text { if } \delta p_{c}=0 \\
0.0 & \text { if } \delta p_{c}<-0.33 \\
1 /\left(2.0 *\left(\delta p_{c}\right)^{2}\right) & \text { if }-0.33 \leq \delta p_{c}<0\end{cases} \end{array}$ \\
\hline drop_food & $\begin{array}{l}\text { Place food around colony nest using a clockwise } \\
\text { search for an empty cell. }\end{array}$ \\
\hline
\end{tabular}

\section{$3 \quad$ Results}

In this section the Ant Foraging Strategy (AFS) is compared with Recursive Spectral Bisection (RSB), Recursive Spectral Bisection plus Kernighan Lin (RSB 
$+\mathrm{KL}$ ) and also Multilevel Kernighan Lin (ML-KL). Each method is tested on two meshes over two, four and eight partitions. Recursive bisection (rb) and simultaneous multiple partitioning (k-way) methods are implemented in the chaco 2.0 package [6]. The number of cuts for the partitions produced are given in Table 2. Mesh 1, (114 nodes, 308 edges), is a two dimensional finite element mesh with triangular elements. Food was placed on an 18 by 18 grid and the algorithm was run for 2000 evaluations. Mesh 2, (286 nodes, 1046 edges), is a two dimensional finite element mesh with quadrilateral elements. The food was placed on a 30 by 30 grid and the algorithm was run for 5000 evaluations. The total number of ants on the grid is always equal to half the total grid area.

Table 2. Number of cuts created by various partitioning methods

\begin{tabular}{|c|l|c|c|c|c|c|c|c|}
\hline Mesh & Method & RSB & RSB & KL+RSB & KL+RSB & ML-KL & ML-KL & AFS \\
\hline & & rb & k-way & rb & k-way & rb & k-way & k-way \\
\hline \hline 1 & Bisection & 25 & 25 & 25 & 25 & 25 & 25 & 25 \\
\hline & Quadrisection & 51 & 51 & 51 & 50 & 50 & 52 & 49 \\
\hline & Octrisection & 89 & 103 & 87 & 97 & 85 & 95 & 80 \\
\hline \hline 2 & Bisection & 29 & 29 & 28 & 28 & 28 & 28 & 28 \\
\hline & Quadrisection & 91 & 91 & 90 & 90 & 88 & 90 & 87 \\
\hline & Octrisection & 176 & 195 & 173 & 191 & 164 & 187 & 161 \\
\hline
\end{tabular}

\section{Discussion and Further Work}

The Ant Foraging Strategy shows some good results on the test meshes. It performs better than RSB, RSB+KL and Ml-KL when implemented for quadrisection and octrisection i.e. simultaneous multiple partitions. It also performs better than RSB and RSB+KL when implemented for recursive bisection. Furthermore, AFS also performs better than recursive multilevel methods in most cases. AFS outperforms RSB because each partition is not dependent on partitions at higher levels of recursion. However, other multiple partitioning schemes perform poorly here. AFS performs well because it provides global structural information (by recursively placing nests) to provide a rough initial partition and a powerful local improvement technique in the form of raiding. AFS is relatively efficient compared to other swarm methods as it maps the the structure onto the environment and places nests to provide a rough initial partition quickly using a small population.

\section{Conclusion}

Most approaches to the k-way partitioning problem use recursive bisection and local improvement techniques, except spectral quadrisection and octrisection 
which perform relatively poorly. AFS provides a novel global method for tackling the problem with built in local improvement which can simultaneously partition into as many sets as required. Results show that swarm-based simultaneous multiple partitioning needs further investigation as it can produce better partitions than recursive bisection techniques which is dependent on the partition found at higher levels of recursion. Furthermore, AFS is relatively efficient compared to other swarm-based methods and scales up well to large meshes [12] when multilevel methods are applied.

\section{References}

1. G. Di Caro and M. Dorigo. Antnet: distributed stigmergic control for communications networks. Journal of Artificial Intelligence Research, 9:317-365, 1998.

2. J.L. Deneubourg, S. Goss, N. Franks, A. Sendova-Franks, C.Detrain, and L. Chretien. The dynamics of collective sorting: Robot like ants and ant-like robots. In First international conference on Simulation of Adaptive Behaviour, July 1990.

3. M. Dorigo and L.M. Garbadella. Ant colonies for the travelling salesman problem. Biosystems, 43:73-81, 1997.

4. L.M. Gambardella, E. Taillard, and M. Dorigo. Ant colonies for the quadratic assignment problem. Journal of Ooperational Research, 50(2):167-176, 1999.

5. M.R. Garey and D.S. Johnson. Computers and Intractability. W.H. Freeman and Company, 1979.

6. B. Hendrickson and R. Leyland. The chaco user's guide, version 2.0. Technical report, Sandia National Laboratories, 1993.

7. B. Hendrickson and R. Leyland. An improved spectral load babancing method. Parallel Processing for Scientific Computing, 1:953-961, 1993.

8. B. Hendrickson and R. Leyland. A multilevel algorithm for partitioning graphs. Technical report, Sandia National Laboratories, 1993.

9. B.W. Kernighan and S. Lin. An efficient heuristic procedure for partitioning graphs. Bell Systems Technical Journal, 49:291-308, 1970.

10. P. Kuntz, P. Layzell, and D. Snyers. A colony of ant-like agents for partitioning in VLSI technology. In Fourth European Conference on Artificial Life, July 1997.

11. P. Kuntz and D. Snyers. Emergent colonization and graph partitioning. In Third International Conference on Simulation of adaptive behaviour: From Animals to Animats 3, July 1994.

12. A.E. Langham and P.W. Grant. A multilevel k-way partitioning algorithm for finite element meshes using competing ant colonies. Technical Report CSR1-99, Dept. of Computer Science, University of Wales Swansea, 1999.

13. H.D. Simon. Partitioning of unstructured problems for parallel processing. Computer Systems in Engineering, 2:135-148, 1991.

14. H.D. Simon and S.H. Teng. How good is recursive bisection? Technical report, NAS Systems Division, NASA,CA, 1993. 